\title{
Lift and Drag on Cylinder of Octagonal Cross-Section in a Turbulent Stream
}

\author{
Md. Jomir Hossain*, Md. Quamrul Islam and Mohammad Ali \\ Dep. of Mechanical Engineering, Faculty of Engineering, Bangladesh University of Engineering \& Technology (BUET), Dhaka-1000, \\ Bangladesh
}

Received 19 May 2013; Accepted 11 December 2013

\begin{abstract}
An experimental investigation of surface static pressure distributions on octagonal cylinder in uniform and turbulent flows was carried out. The study was performed on both the single cylinder and the group of two cylinders, two cylinders were used, one was at the upstream side, and the other was at the downstream side of the flow. They were placed centrally along the flow direction. The inter-spacing space between the two cylinders was varied at $1 \mathrm{D}, 2 \mathrm{D}, 3 \mathrm{D}, 4 \mathrm{D}, 5 \mathrm{D}$, $6 \mathrm{D}, 7 \mathrm{D}$ and $8 \mathrm{D}$, where $\mathrm{D}$ is the width of the cylinder across the flow direction. The pressure coefficients were calculated from the measured values of the surface static pressure distribution on the cylinder. Then the drag and lift coefficients were obtained from the pressure coefficients by the numerical integration method. It was observed that at various angles of attack, the values of the lift coefficients and drag coefficients were insignificant compared to those for a sharp-edged square cylinder. The strength of the vortex shedding was shown to be reduced as the intensity of the incident turbulence was increased. Measurements of drag at various angles of attack $\left(0^{\circ}\right.$ to $\left.40^{\circ}\right)$ showed that with increase in turbulence level the minimum drag occurred at smaller values of angle of attack.
\end{abstract}

Keywords: Octagonal cylinder, Drag coefficients, Lift coefficients, Tall building, Turbulent Stream

\section{Introduction}

In the $17^{\text {th }}$ century, Galileo and Newton have considered the effect of wind loading on buildings, but during that period, it did not gain popularity. The effect of wind loading on buildings and structures has been considered for design purposes since late in the 19th century; but starting from that time up to about 1950 , the studies in this field have not been considered seriously. Building and their components are to be designed to withstand the code specified wind loads. Calculating wind loads is important in the design of wind force resisting system, including structural members, components, and cladding against shear, sliding, overturning and uplift actions.

In recent years, much emphasis has been given on "The study of wind effect on buildings and structures" in the different corners of the world. Even researchers in Bangladesh have taken much interest in this field. Till now, little attention has been paid to the flow over the bluff bodies like square cylinders, rectangular cylinders, hexagonal cylinders etc. and some information is available concerning the flow over them in staggered condition, although this is a problem of considerable practical significance. With the progressing world, Engineering problems regarding wind loads around a group of skyscrapers, chimneys, towers and the flow induced vibration of tubes in heat exchangers, bridges, oil rigs or marine structures need detailed investigation of flow patterns and aerodynamic

* E-mail address: jomirlal@yahoo.com characteristics. Arising from the increasing practical importance of bluff body aerodynamics, over the past few decades' sufficient effort has been given in research works concerning laboratory simulations, full-scale measurements and more recently numerical calculations and theoretical predictions for flows over bodies of wide variety of shapes. A number of failures of bridges, transmission towers, buildings and housings over the last one hundred years prompted researchers to do research work in this field.

It is the great challenge of the engineers and architects to reduce the wind load on the tall buildings. Now a day due to huge population pressure, emphasis on design and construction of the tall buildings is being given in many places. Especially the design of the group of tall buildings is the most important consideration to take care of the housing problem of the huge population. As the building becomes tall it is necessary to take into consideration the effect of wind on its design. Keeping this in mind the study on the group of octagonal cylinders has been conducted, which will be applicable to obtain the wind load on the group of tall buildings. The study of wind effect was first limited to loading on buildings and structures only, possibly because of its most dramatic effects are seen in their collapses. In midsixties, researchers started the study of less dramatic, but equally important environmental aspects of flow of wind around buildings. These include the effects on pedestrians, weathering, rain penetration, ventilation, heat loss, wind noise and air pollution etc. The pioneer researcher in this field is Iawson $T V{ }^{[1]}$ of the IIniversity of Rristol A 
number of works of the environmental aspects of wind was being studied at the Building Research Establishment at Garson and the University of Bristol, UK.

It is true that researchers from all over the world have contributed greatly to the knowledge of flow over bluff bodies as published by Mchuri, F. G. ${ }^{[2]}$ but the major part of the reported works are of fundamental nature involving the flow over single body of different profiles. Most of the researchers have conducted works either on single cylinder with circular, square, hexagonal or rectangular sections etc. or in a group with them for various flow parameters. However, the flow over octagonal cylinders has not been studied extensively especially in-groups to date, although this is a problem of practical significance. It is believed that the study on the cylinder with octagonal section will contribute to find the wind load on the single and group of octagonal buildings and the results will be useful to the relevant engineers and architects. There are various parameters, which control the flow behaviour as mentioned by Castro, J.P. ${ }^{[3]}$. They are (i) vortices in front of the building, (ii) opening through buildings, (iii) spacing of rows, (iv) wakes of buildings, (v) long straight streets, (vi) narrowing streets, (vii) corners and (viii) courtyards. The mean wind speed varies with height. The variation of wind speed has been expressed by Davenport, A. C. ${ }^{[4]}$. The wind in the atmospheric boundary layer varies in time and space. The source of wind energy is the sun that emits solar radiation, which causes differential heating of the earth surface and the atmosphere.

In the atmosphere there is a general convective transport of heat from lower to higher latitudes in order to make the earth's radiation imbalance as mentioned by Lanoville, A. ${ }^{[5]}$. It is for this reason that the atmosphere is a restless medium in which circulation of all sizes is normal. Lee (6) performed study on the effect of turbulence on the surface pressure field on a square prism. In his study measurements were presented of the mean and fluctuating pressure field acting on a two dimensional square cylinder in uniform and turbulent flows. In his investigation he showed that the addition of turbulence reduces the drag on the cylinder. Mandal and Farok ${ }^{[7]}$ measured the static pressure distributions on the single cylinder with square and rectangular cross-section having rounded corners in a uniform cross flow. The experiment was conducted for different corner radii and side dimensions of the cylinders at zero angle of attack. The experimental results reveal that the corner radius of the cylinder has significant effect while the side dimension has some effect on the drag coefficient.

It is the task of the engineer to ensure that the performance of structures subjected to the action of wind will be adequate during their anticipated life from the standpoint of both structural safety and serviceability. To achieve this end, the designer needs information regarding (i) the wind environment, (ii) the relation between that environment and the forces it induces on the structures, (iii) the behaviour of the structure under the action of forces. The knowledge of wind loading on a single tall building or on a group of tall buildings is essential for their economic design. The flow around an octagonal model cylinder can be ideally considered analogous to that of the flow around a tall octagonal-shaped building. Therefore, a study of wind flow around groups of octagonal cylinders would be helpful in this respect. For designing groups of tall buildings, knowledge of the effect of wind loading on a single tall building is not sufficient because the effects of nearby buildings on the loads imposed on a structure would be quite different. The wind in the atmospheric boundary layer varies in time and space. It depends on the terrain roughness, the local wind climate and on variations in temperature. Usually the effects of temperature are assumed negligible, when studying wind loads relevant are the proper simulation of the wind speed with height and the turbulent characteristics.

The wind tunnel testing provides information regarding the dependence of particular response parameters on wind speed and direction. In order to make the most rational use of this aerodynamic information, it is necessary to synthesize it with the actual wind climate characteristics at the site. The characteristics necessary to define are those governing wind speed and direction at a suitable height above ground level at the site. The joint probability distribution of wind speed and direction then defines the wind climate, whereas all of the aerodynamic information, which includes sensitivities to building orientation and to its surroundings, is contained in the wind tunnel data. Though the problem regarding the wind loadings on buildings and structures is common to all parts of the world and it is expected that the solution will not be significantly different from country to country, yet research work should be carried out in this field considering the climatic conditions and problem of this country so that a clear picture about the nature of wind loading can be obtained. The data from these research works should enable to the architects, engineers and town planners of Bangladesh to design buildings and structures more efficiently.

The scale difference between wind tunnel model and prototype is found in the high frequency fluctuation. High peaks found on the cladding in full-scale are not found in the wind tunnel. Those effects may be caused by structural details that are not simulated in the wind tunnel model. Now-a-days, both the studies with models and full-scale buildings are being performed to compare the result for varying the validity of the former. But full-scale experiments are both costly and difficult to perform. For the present study with staggered buildings full- scale experiments will not only be complex and costly but it would be difficult to record reliable pressure distribution simultaneously on the group of buildings as there will be variation of speeds and direction of wind with time.

\section{Experimental set-up}

\subsection{Wind Tunnel}

The test was conducted at the exit end of an open circuit subsonic wind tunnel. In Figure 1 the schematic diagram of the wind tunnel is presented showing the position of the cylinders at the exit end of the wind tunnel. One cylinder was located at the upstream side called front cylinder and the other one was located at the downstream side called rear cylinder. The wind tunnel was $6 \mathrm{~m}$ long with a test section of $460 \mathrm{~mm} \times 460 \mathrm{~mm}$ cross section. The cylinders were fixed to the side walls of the extended portion at the exit end.

In the side wall the cylinder was fastened rigidly at one end and through the other end of the cylinder the plastic tubes from the tapping were taken out and were connected with the inclined multi-manometer, which contained water as the manometer liquid. The cylinder was leveled in such a way that the flow direction was parallel to its top and bottom sides and perpendicular to the front side. The axis of the cylinder was at the same level to that of the wind tunnel. To generate the wind velocity, two axial flow fans are used. Each of the fans is connected with the motor of 2.25 kilowatt 
and $2900 \mathrm{rpm}$. The induced flow through the wind tunnel is produced by two-stage rotating axial flow fan of capacity $18.16 \mathrm{~m}^{3} / \mathrm{s}$ at the head of $152.4 \mathrm{~mm}$ of water and $1475 \mathrm{rpm}$.

In each case of the tests, wind velocity is measured directly with the help of a digital anemometer and the flow velocity in the test section was maintained at $13.2 \mathrm{~m} / \mathrm{s}$ approximately. The measured velocity distribution was almost uniform across the tunnel test section in the upstream side of the test models.

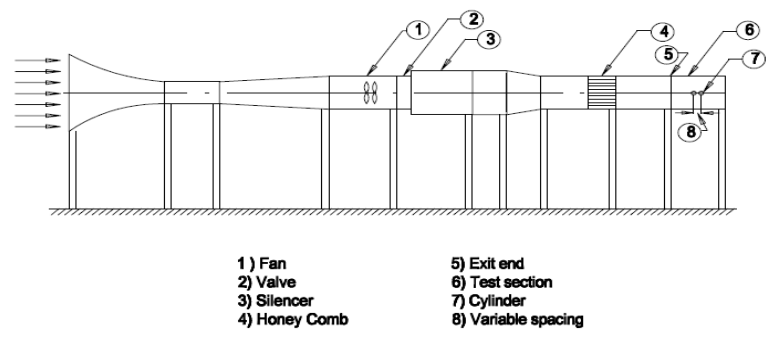

Fig. 1. Schematic diagram of wind tunnel

There was a provision for rotation of the test cylinder at various angles to obtain the wind load at different angles of attack. The Reynolds number was $4.13 \times 10^{4}$ based on the projected width of the cylinder across the flow direction. Since the top and bottom of the extended part of the wind tunnel was open; as such no correction for blockage was done in the analysis. The test cylinders were placed very close to the end of the wind tunnel so that the approach velocity on the test cylinders was approximately identical as that in the exit end of the wind tunnel.

\subsection{Constructional Details of Cylinders}

The tapping positions on the cross-section of the cylinder are shown in Figure 2. The width of the octagonal cylinder was $50 \mathrm{~mm}$ as shown in this figure. Each face of the cylinder contained five tappings.

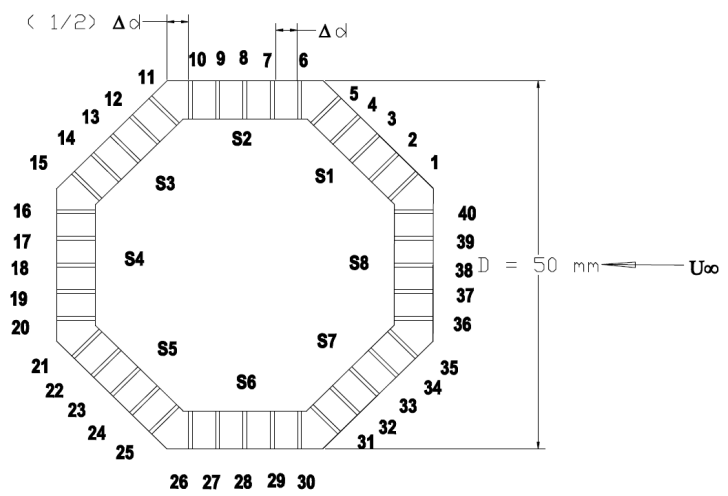

Fig. 2. Tapping positions shown on cross-section of cylinder

In Figure 3 the tapping positions on the longitudinal section of the cylinder is shown. There were five tappings on each face of the cylinder. The distance between the consecutive tapping points was equal $(\Delta d)$ as shown in the figure. However, the location of the corner tapping was at a distance of $1 / 2 \Delta \mathrm{d}$. Each taping was identified by a numerical number from 1 to 40 as can be seen from the figure. It can be seen from the longitudinal section that the tappings were not made along the cross-section of the cylinder.

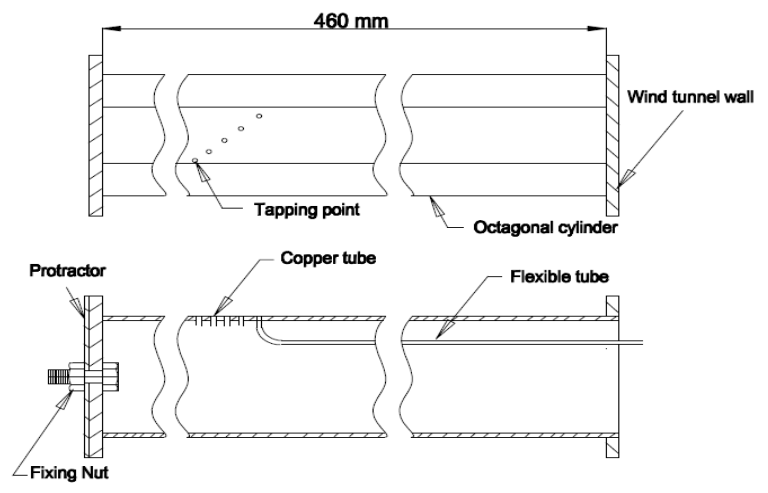

Fig. 3. Tapping positions shown on longitudinal section of cylinder

They were located within some span of the cylinder as shown in Figure 3. On one side of the cylinder a steel plate was attached through which there was a bolt for fixing the cylinder with the side wall of the extended tunnel as shown in Figure 3. The other side of the cylinder was hollow through which the plastic tubes were allowed to pass. The plastic tubes were connected with the copper capillary tubes at one side and at the other side with the inclined multimanometer. The manometer liquid was water. The tappings were made of copper tubes of $1.71 \mathrm{~mm}$ outside diameter. Each tapping was of $10 \mathrm{~mm}$ length approximately. From the end of the copper tube flexible plastic tube of $1.70 \mathrm{~mm}$ inner diameter was press fitted.

\subsection{Single Cylinder}

The upstream velocity was assumed to be uniform and the flow occurred across the cylinder. In Figure 4 the position of the single cylinder at zero angle of attack is shown in the wind tunnel test section.

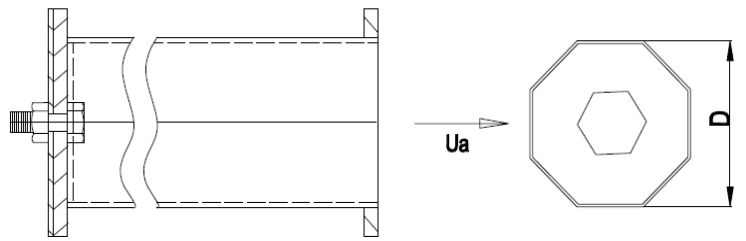

Fig. 4. Tunnel test section showing position of single cylinder

The surface static pressure distributions on eight faces of the cylinder were measured in this position. Then the cylinder was rotated at an angle of $10^{\circ}$ and the static pressure distributions on each face of the cylinder were measured again. The same test procedure was repeated to measure the surface static pressure distributions of the cylinders with angles of attack of $0^{\circ}, 10^{\circ}, 20^{\circ}, 30^{\circ}, 40^{\circ}$ and $50^{\circ}$

2.4. Cylinders in Group

They were placed centrally along the flow direction. In Figure 5 the position of the group of cylinders at zero angle of attack is shown in the wind tunnel test section.
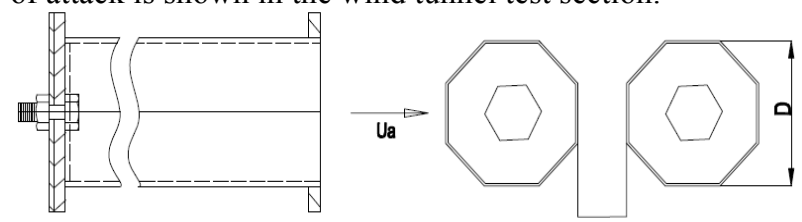

Inter-Spacing

Fig. 5. Tunnel test section showing position of group cylinders

The inter-spacing between the front cylinder and the rear crylinder vxas taken as $1 \mathrm{C}$ i e $50 \mathrm{~mm}$ Then static curface 
pressure distributions were measured on the eight faces of the front and the rear cylinders. Keeping everything identical the inter-spacing was changed to $2 \mathrm{D}$ and the experiment was repeated. Next, the inter-spacing was varied to $3 \mathrm{D}, 4 \mathrm{D}, 5 \mathrm{D}$, $6 \mathrm{D}, 7 \mathrm{D}$ and $8 \mathrm{D}$ and in each case the static pressure distributions on both the front and the rear cylinders were taken.

\section{Mathematical Model}

The pressure coefficient is defined as

$$
C_{P}=\frac{\Delta \mathrm{P}}{\frac{1}{2} \rho u_{\infty}^{2}}
$$

Drag and lift coefficients are defined as follows

$$
C_{D}=\frac{F_{D}}{\frac{1}{2} \rho u_{\infty}^{2}}
$$

and

$$
C_{L}=\frac{F_{L}}{\frac{1}{2} \rho u_{\infty}^{2}}
$$

The detailed calculation of $C_{P}$ can be found in Mandal, A. C. [7]

\section{Result and Discussion}

\subsection{General}

The distributions of the static pressure coefficients-on the surface of the cylinders in group for various inter-spacing are considered. In case of the group of cylinders at zero degree angle of attack, the lift coefficient was zero due to symmetry. The results of the present research work have been compared with those of the existing research works in some cases.

\subsection{Single Cylinder}

The distributions of the pressure coefficients drag and lift coefficients have been taken into consideration for discussion for a single octagonal cylinder at different angles of attack. Then the drag and lift coefficients have been obtained from the pressure coefficients by the numerical integration method.

\subsection{Distribution of Pressure Coefficients}

The cross-section of the single octagonal model cylinder with 40 numbers of tappings, eight numbers on each surface of the cylinder at an angle of attack has been shown in Figure 6. The eight surfaces have been identified with $\mathrm{S}_{1}, \mathrm{~S}_{2}$, $\mathrm{S}_{3}, \mathrm{~S}_{4}, \mathrm{~S}_{5}, \mathrm{~S}_{6}, \mathrm{~S}_{7}$ and $\mathrm{S}_{8}$. Pressure coefficient for each taping point has been determined from the measured surface static pressure. In Figure 7, the distributions of static pressure coefficients for angles of attack of $0^{\circ}$ to $40^{\circ}$ with a step of $10^{\circ}$ have been presented respectively. While in Figure 7, the distributions of pressure coefficients for all angles of attack have been shown for relative comparison.

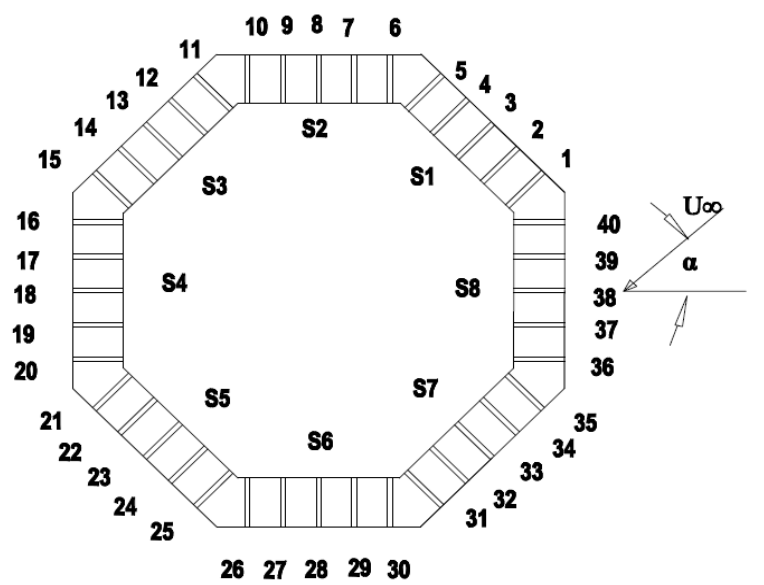

Fig. 6. Flow over single cylinder at an angle of attack

\subsection{Variation of Drag Coefficient}

Variation of drag coefficient at various angles of attack on single octagonal cylinder is shown in Figure 8. The drag coefficient at different angles of attack on a single square cylinder at uniform flow obtained by Mandal, A. C. [7] is also presented in this figure for comparison. It can be noticed from this figure that there is significant drop in the drag coefficient values for the octagonal cylinder in comparison to that of the square cylinder and the values approach to that of the circular cylinder. It is seen from this figure that at zero angle of attack, the drag coefficient is about 0.99 and at all other angles of attack, the values are close to 0.90 except at angle of attack of $10^{\circ}$, where the value is about 0.60 . The values of the drag coefficient at various angles of attack for the octagonal cylinder can be explained from the $\mathrm{Cp}$-distribution curves.

\subsection{Variation of Lift Coefficient}

In Figure 9 the variation of lift coefficient at various angles of attack on single octagonal cylinder is shown. The lift coefficient at different angles of attack on a square cylinder at uniform flow obtained by Mandal, A. C. [7] is also presented in this figure for comparison. It can be noticed from this figure that the variation of the lift coefficient on the single octagonal cylinder is not appreciable; they are close to zero value except at angles of attack of $10^{\circ}$ and $40^{\circ}$, where some insignificant values are observed. For the single square cylinder the variation of lift coefficient with angle of attack is remarkable. The values of the lift coefficients for the single octagonal cylinder can be explained from the $\mathrm{Cp}$ distribution curves.

\subsection{Group of Cylinders}

In Figure10, the group of cylinders is shown at zero angle of attack. One cylinder is positioned in the upstream side designated as the front cylinder and another one is positioned in the downstream side designated as the rear cylinder. Both of them are placed along the flow direction. The inter-spacing between them will only be varied at $1 \mathrm{D}$, 2D, 3D, 4D, 5D, 6D, 7D and 8D keeping the angle of attack constant. Tapping points are shown along the cross-section on the eight surfaces $\mathrm{S}_{1}, \mathrm{~S}_{2}, \mathrm{~S}_{3}, \mathrm{~S}_{4}, \mathrm{~S}_{5}, \mathrm{~S}_{6}, \mathrm{~S}_{7}$ and $\mathrm{S}_{8}$ of the cylinder, where the surface static pressures were measured. 


\subsection{Variation of Drag Coefficient on Front and Rear Cylinder}

The variation of drag coefficients on the front and rear cylinders of the group at different inter-spacing at zero degree angle of attack has been presented in Figure 11. It can be seen from this figure that the drag coefficient on the front cylinder is about unity. Except at the inter-spacing of $1 \mathrm{D}$, at all other inter-spacing the drag coefficients on the front cylinder of the group are higher than that on the single octagonal cylinder. That is, due to the interference of the flow by the rear cylinder, there has been increase of the drag values on the front cylinder of the group at all inter-spacing except at the inter-spacing of $1 \mathrm{D}$ in comparison to the single octagonal cylinder. However, there has been remarkable drop in the drag value on the rear cylinder of the group. From Figure 13 it is observed that at some inter-spacing between $2 \mathrm{D}$ and $3 \mathrm{D}$, the drag coefficient on the rear cylinder is zero. At the higher inter-spacing the drag coefficients are positive and at lower inter-spacing they are negative. The drag coefficient on the rear cylinder of the group drops mainly because the front surfaces $S_{1}$ and $S_{8}$ of the rear cylinder fall within the suction side generated by the front cylinder of the group.

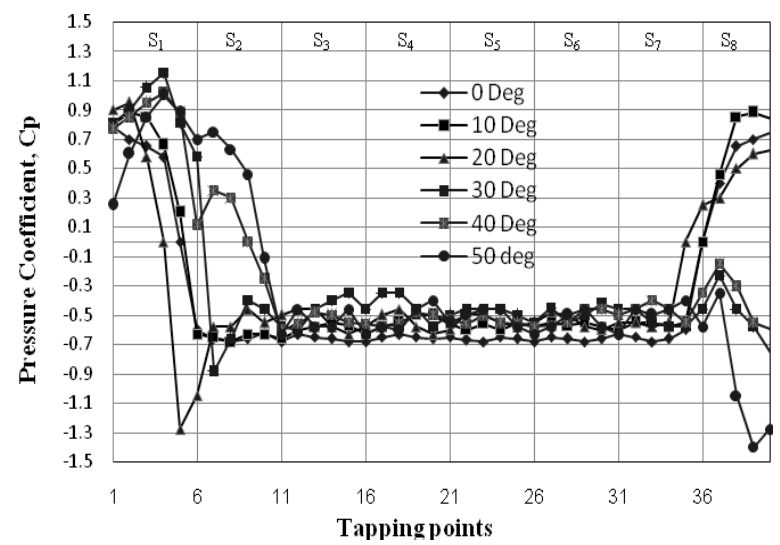

Fig. 7. Distribution of pressure coefficient at different angles attack

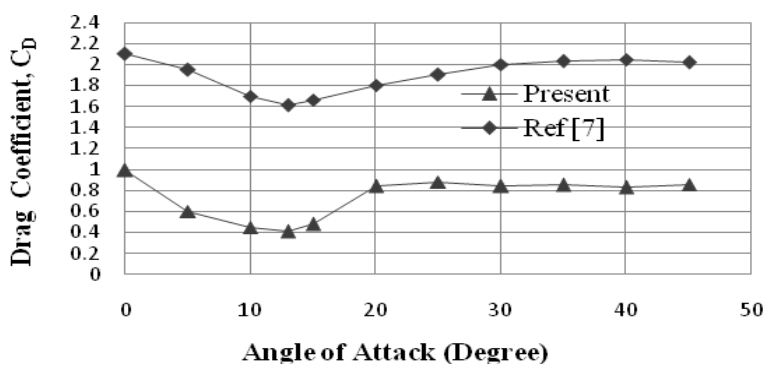

Fig. 8. Variation of drag coefficient at various angles of attack on single cylinder

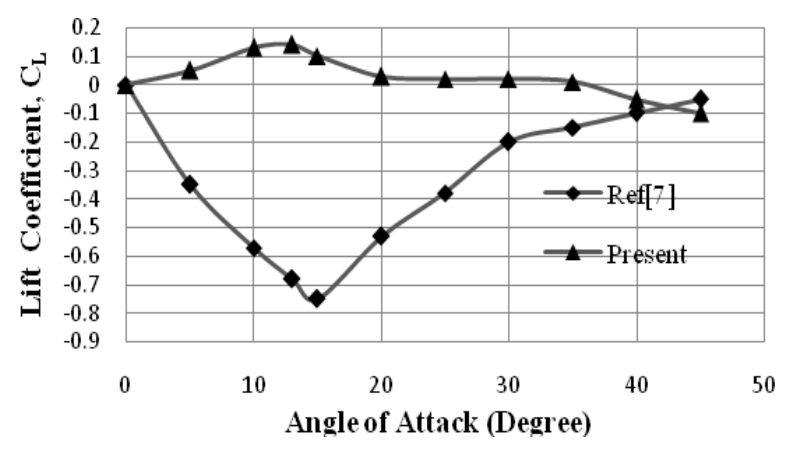

Fig. 9. Variation of lift coefficient at various angles of attack on single cylinder

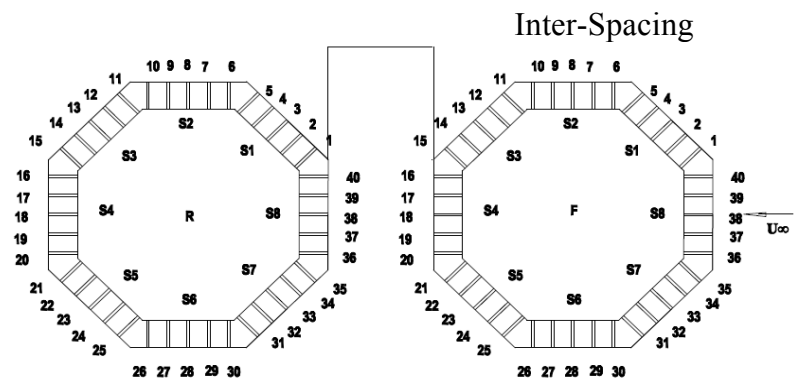

Fig. 10. Flow over cylinder in group at zero angle of attack

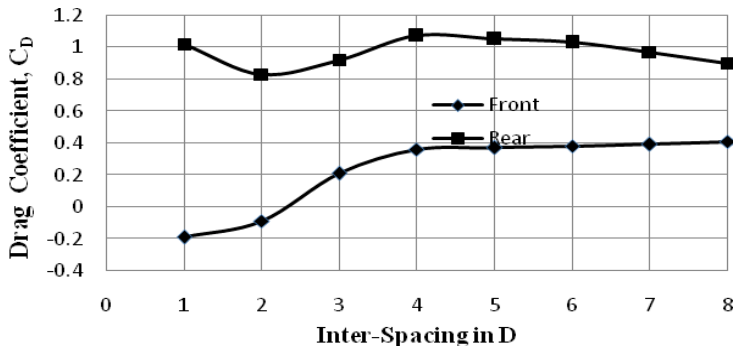

Fig. 11. Variation of drag coefficient on front $\&$ rear cylinders at different inter-spacing

\section{Conclusions}

The drag coefficient for a single octagonal cylinder at zero angle of attack is about 0.95 in contrast to that of 2.0 for a single square cylinder at the same angle of attack. There is significant drop in the drag coefficient values for the single octagonal cylinder in comparison to that of the single square cylinder and the values approaches to that of the circular cylinder. The variation of the lift coefficient on the single octagonal cylinder is not appreciable and they are close to zero value except at angles of attack of $10^{\circ}$ and $40^{\circ}$, where some insignificant values are observed. There is small change of drag coefficient on the front cylinder compared to that on the single cylinder. There has been remarkable drop in the drag coefficient on the rear cylinder of the group as it falls in the suction region generated by the front cylinder. Except at the inter-spacing of 1D, at all other inter-spacing the drag coefficients on the front cylinder of the group are higher than that on the single octagonal cylinder. It is observed that at some inter-spacing between 2D and 3D, the drag coefficient on the rear cylinder is zero. At the higher inter-spacing the drag coefficients are positive and at lower inter-spacing they are negative.

\section{Acknowledgements}

This research work was funded by Department of Mechanical Engineering, Bangladesh University of Engineering \& Technology (BUET).

$\begin{array}{cl}\text { Nomenclature } & \\ A & \text { Frontal area of the Cylinder } \\ \text { I } & \text { Net force } \\ F_{D} & \text { Drag force } \\ F_{L} & \text { Lift force } \\ \text { C } & \text { Cnefficient } \mathrm{nf} \mathrm{lift}\end{array}$


Md. Jomir Hossain, Md. Quamrul Islam and Mohammad Ali /Journal of Engineering Science and Technology Review 6 (3) (2013) 105-110

$\begin{array}{llcc}\mathrm{C}_{\mathrm{D}} & \text { Coefficient of drag } & \omega & \text { Angular velocity of the earth } \\ \mathrm{C}_{\mathrm{p}} & \text { Coefficient of pressure } & \mathrm{X} & \text { Latitude velocity of the earth } \\ \mathrm{P} & \text { Static pressure on the surface of the cylinder } & \Delta \mathrm{P} & \text { Pressure difference } \\ \mathrm{P}_{\mathrm{o}} & \text { Ambient pressure } & \Delta \mathrm{h}_{\mathrm{w}} & \text { Manometer reading } \\ \rho & \text { Density of the air } & \gamma_{\mathrm{w}} & \text { Specific weight of manometer liquid (water) } \\ \mathrm{U}_{\infty} & \text { Free stream velocity } & \mathrm{h}_{\mathrm{a}} & \text { Air head } \\ \mathrm{V} & \text { Wind speed } & \gamma_{\mathrm{a}} & \text { Specific weight of air } \\ \mathrm{Z} & \text { Height } & \alpha & \text { Angle of attack } \\ \mathrm{dp} / \mathrm{dn} & \text { Pressure gradient } & & \end{array}$

\section{References}

1. J. T.V. Lawson, "Wind loading of buildings, possibilities from a wind Tunnel investigation", University of Bristol, U.K. Report on TVL /731A, August, 1975.

2. F.G. Mchuri, "Effects of the free stream turbulences on drag coefficients of bluff sharp- edged cylinders", Nature, Vol.224, No.5222, November 29, 1969.

3. J.P. Castro, and J.E. Fackwell, "A note on two- dimensional fence flows with emphasis on wall constant", J. Industrial. Aerodynamic, 3(1), March1978.

4. A.G. Davenport, "The relation to wind structure to wind loading" "Proceedings of the conference on wind effects on buildings and structures", Vol.1, June, 1963.

5. A. Lanoville, I.S. Gateshore, and G.V. Parkinsoon, "An experimental of some effects of turbulence on bluff bodies", Proceeding of the 4th international conference on wind effects on buildings and structure, London, U.K.1975, pp.333-341.

6. B.E. Lee, "The Effect of Turbulence on the Surface Pressure Field of a Square Prism", Journal of Fluid Mechanics, Vol.6 J.E. 9, Part 2, 1975, pp. 263-282.

7. A.C. Mandal, and G.M.G. Farok, "An experimental investigation of static pressure distributions on a group of square and rectangular cylinders with rounded corners", Submitted for publication in the Journal of Mechanical Engineering, The Institution of Engineers, Bangladesh 